\title{
EL YO FRACTURADO: Sensación de agencia y psicopatología
}

\author{
Luis Moisés López Flores \\ Instituto Tecnológico y de Estudios Superiores de Monterrey \\ (ITESM), México
}

Resumen: Tras los experimentos de B. Libet, la discusión contemporánea sobre la libertad y el determinismo ha inclinado la balanza hacia este último. El avance de las neurocienciasha reenfocado la discusión antes centrada en la idea de libertad hacia el concepto de "sensación de agencia" (SA) y con ello el trabajo de la psicopatología de la acción ha sido un terreno fructífero para dicha querella. En la presente investigación sostengo que algunos casos de disrupciones en la agencia, como algunas patologías psicológicas y motoras, pueden servir como evidencia para SA. $\mathrm{Al}$ final de la investiǵación revisaré alǵunas consecuencias normativas del SA.

Palabras clave: psicopatología filosófica, sentido de aǵencia, libertad y determinismo, filosofía de la psiquiatría

The Broken Self: Sense of Agency and Psychopathology

Abstract: After B. Libet's experiments determinism has taken thecore position against free will believers. The development of neuroscience has refocused the debate from the old-term freedom to the concept of "sense of agency" (SA). With this turn in mind the work in the psychopathology of action has been a fruitful field for that long-standing discussion. In this paper I argue that some disruptions of agency such as some motor and psychological pathologies can work as evidence for the SA. At the end of the paper I will examine some normative consequences for SA.

Keywords: philosophical psychopathology, sense of agency, freedom and determinism, philosophy of psychiatrics.

Recibido: 22/02/2018. Aprobado: 20/07/2018.

\section{Introducción}

De acuerdo con algunos autores la discusión sobre libertad y determinismo es quizá la problemática más debatida en la historia de la 
filosofía. ${ }^{1}$ El conflicto nace de una doble conciencia, desde el punto de vista personal nos vemos como seres libres capaces de influir en el mundo, desde un punto de vista impersonal nos concebimos como seres cuyas acciones son causadas por factores externos sobre los cuales no tenemos control. El determinismo pese a su variedad (fatalista, teológico, físico, psicológico o lógico) contiene una noción central por la cual establece un amenaza al libre albedrío, a saber, la existencia de condiciones previas suficientes para la ocurrencia de un evento, de tal modo que si estas condiciones se cumplen, el evento en cuestión se debe cumplir. ${ }^{2}$

A reserva de ampliar la discusión al final de la investigación sólo haré algunos señalamientos. Los debates contemporáneos en la línea determinista han virado de las discusiones en las ciencias físicas hacia otras ciencias (biología, ciencias cognitivas, ciencias sociales). Es de especial interés el auǵe de las neurociencias. La evidencia reinante que provee la neurociencia de la acción no sólo ha engrosado las filas que apoyan de manera entusiasta un cierto tipo de determinismo, sino que ha disminuido la confianza en soluciones compatibilistas. ${ }^{3}$ Dicho entusiasmo no es tan novedoso en su espíritu como sí lo es en la cantidad de investigaciones al respecto del cómo, cuándo y dónde aparece la voluntad consciente. ${ }^{4}$

\footnotetext{
${ }^{1}$ Para un recuento, véase, Kane, R. The Oxford Handbook of Free Will. New York: Oxford University Press, 2002.

${ }^{2}$ Kane, R. op. eit., pp. 5 y ss.

${ }^{3}$ Véase, Bickle, J. [en línea]: "The Philosophy of Neuroscience" enThe Stanford Encyclopedia of Philosophy, 2012, Edward N. Zalta (ed.), URL = http://plato.stanford. edu/archives/sum2012/entries/neuroscience/, [Consultado: 30/06/2017]; Kane, R. op. cit.; Racine, E. Pragmatic Neuroethics. Improving Treatment and Understanding of the Mind-Brain. London: MIT Press, 2010.

${ }^{4}$ Bickle, J. op. cit., señala muy bien el entusiasmo naturalista del funcionalismo anǵlosajón de los 50-70as (Feigl, Smart, Putnam) como un antecedente del eliminativismo de Churchland, P. Brain Trust. What Neuroscience Tell Us about Morality. Princeton: Princeton, 2011. Véase, Kim, J. Mind in a Physical World. An Essay on the Mind-Body Problem and Mental Causation. Cambridge: MIT Press, 2000, pp. 5 y ss.; Frit, C \& Rees, G. «A Brief History of the Scientific Approach to the Study of Consciousness» y M. Tye, M. «Philosophical Problems of Consciousness» ambos en Velmans, M. The Blackwell Companion to Consciousness. Oxford: Blackwell Publishing, 2007; cf. Glock, H. What
} 


\section{El yo fracturado}

En 1985 en un famoso experimento, Benjamin Libet mostraba que antes de que el agente tomara conciencia de su intención ya existía un proceso cerebral inconsciente de iniciación llamado potencial de preparación (readinesspotential $[\mathrm{RP}]$ ), es decir, que existía evidencia de actividad cerebral no sólo pre-motora, sino pre-intencional (350-400 ms después del RP y 200 ms antes del acto motor). Por tanto, la función (o al menos una) que el agente desempeña no es la que tradicionalmente se le adjudica, a saber, la de iniciar la acción voluntaria, sino la de seleccionar y controlar las acciones. ${ }^{5}$ ¿Cómo entender estos descubrimientos? La postura más usual interpreta los resultados del experimento como evidencia de la carencia de fundamentos para conceptos como el de libertad, voluntad consciente, agencia etc., es decir, la estrategia promete resolver de una vez por todas la pugna entre determinismo y libertad inclinando la balanza del lado del primero. ${ }^{6}$

Sin embargo, tanto el experimento como la interpretación de los resultados del mismo han levantado objeciones no sólo por parte de filósofos con filiaciones dualistas o compatibilistas, sino por neurocientíficos y psicólogos quienes consideran problemáticos ciertos presupuestos conceptuales, fenomenológicos y experimentales. ${ }^{7}$ Una línea de investigación reciente sugiere que el problema entre libertad y determinismo se puede resolver a partir de la diferencia entre "sensación de agencia" $(\mathrm{SA})^{8}$ y "conciencia de la acción" (CA), esto es,

is Analytic Philosophy? Cambridge: Cambridge, 2008, pp. 134-148: Block, N. Consciousness, Function, and Representation. Collected Papers. Vol. I, Cambridge: MIT Press, 2007

${ }^{5}$ Libet, B. "Unconscious Cerebral Initiative and the Role of Conscious Will in Voluntary Action" en The Behavioral and Brain Sciences, 8, 1985, pp.529-566; igualmente, "Do We Have Free Will?" en Sinnott-Armstrong, W. \& Nadel, L. Conscious Will and Responsibility. Oxford: Oxford, 2011.

${ }^{6}$ Nadelhoffer, T. "The Threat of Shrinking Agency and Free Will Disillusionism" enSinnott-Armstrong, W. \& Nadel, L. op. cit.; ef. Wegner, D. The Illusion of Conscious Will. Cambridge: MIT Press, 2002; Haggard, P. "Conscious Awareness of Intention and of Action" en Roessler J. \& Elian N. Agency and Self-awareness: Issues in Philosophy and Psychology. Oxford: Oxford University Press, 2003.

${ }^{7}$ Véanse los comentarios finales de Libet, B. Unconscious Cerebral cit., pp. 539-564; el compendio de Sinnott-Armstrong, W. \& Nadel, L. op. cit.

${ }^{8}$ Usaré SA tanto para "sensación" como "sentido" de aǵencia 
existe una diferencia entre sentir que uno es el autor de las acciones, ser su dueño (ownership) y estar meramente consciente de ellas. La distinción suele ser trazada a partir no sólo de un criterio conceptual (algo que ya ha hecho desde hace mucho la filosofía de la mente y la acción) ${ }^{9}$, sino desde un criterio fenomenológico y psicológico (cómo y por qué experimentamos el contenido de cada una de las experiencias mencionadas). Dicha distinción es discutida dentro de una teoría neurológica de la acción. ${ }^{10}$

En esta segunda aproximación la evidencia más precisa (más no la única) es extraída sobre todo de la llamada "filosofía de la psicopatología". "La filosofía de la psicopatología incluye tanto el estudio de los temas filosóficos que emergen de los desórdenes mentales, como los problemas metateóricos que surgen del ejercicio clínico y la investigación científico-médica. ${ }^{12}$ En nuestro caso nos limitaremos a la primera parte en lo que concierne a las "disrupciones en el sentido de la agencia". Las disrupciones pueden ser desde fallas en la percepción y propiocepción, fallas en la atención, hasta fallas más generales como trastornos de la personalidad. Por ende, las patologías corren desde

9 Bratman, M. Intention, Plans, and Practical Reason. Cambridge: MA: Harvard University Press, 1987; Davidson, D. Essays on Actions and Events, Oxford. Clarendon Press: 1980; Frankfurt, H. The Importance of What We Care About. Cambridge: Cambridge University Press, 1988

${ }^{10}$ Balconi, M. (ed.). Neuropsychology of the Sense of Agency. From Consciousness to Action. Milán: Springer, 2010; Roessler J. \& Elian. op. cit.; Sinnott-Armstrong, W. \& Nadel, L. op. cit.;Young, Garry, Philosophical Psychopathology. Philosophy without Thought Experiments. London: Palgrave Macmillan, 2013

${ }^{11}$ Graham, G. The Disordered Mind: An Introduction to Philosophy of Mind and Mental Illness. London: Routledge, 2010; Graham, G. \& Stephens L. (eds.). Philosophical Psychopathology. Cambridge, MA: MIT Press, 1994; Young, G. op. cit.; Roceo, G. Disturbed Consciousness. New Essays on Psychopathology and Theories of Consciousness. London: The MIT Press, 2015.

${ }^{12}$ Graham, G. \& Stephens L. op. cit., pp. 4-6. Graham señala que esta división se puede entender también como psicopatología y filosofía de la mente aplicada; psicopatología y ciencia de la mente; y psicopatología y ética (pp. 6-18). Por su parte Young, op. cit., señala la pertinencia metodológica de las psicopatologías frente a los experimentos mentales usados en filosofía de la mente; Dennett, D. ElbowRoom: The Varieties of Free Will Worth Having. Cambridge. MA: MIT Press, 1984. 


\section{El yo fracturado}

un síndrome de la mano anárquica, síndrome de la mano extraña, negación de atención, hasta esquizofrenia, trastorno obsesivo compulsivo, autismo, pseudo-crisis, etc. ${ }^{13}$

En muchos casos lo que podemos observar es una distinción fenomenológica entre la CA y SA en diversos grados. El esquizofrénico, por ejemplo, está consciente de la acción, incluso de las intenciones que conforman la acción (relación causal de intenciones y movimientos) pero niega la propiedad de las intenciones (la llamada inserción de intenciones). En el síndrome de la mano anárquica el agente está consciente de las acciones calculadas y bien ejecutadas de su mano anárquica, pero desconoce el contenido intencional de las mismas, y por ende, se disocia de su propiedad.

En la presente investigación intentaré defender la necesidad de la agencia a partir de la distinción fenomenológica deSA (en particular el síndrome de la mano anárquica, trastorno obsesivo compulsivo y la esquizofrenia). El SA se justifica por motivos prácticos y de psicología del sentido común. Así, el plan de trabajo es el siguiente: describiré brevemente el experimento de Libet y las consecuencias negativas para la aǵencia [1]. Presentaré algunas objeciones a dichas consecuencias a partir de algunos casos patológicos que sugieren la existencia de la distinción entre SA y CA [2]. Responderé a las objeciones del punto anterior mediante la teoría de ilusión de la agencia de Daniel Wegner [3]. Y finalmente revisaré algunas consecuencias normativas [4].

\section{2. ¿Evidencia en contra de la agencia?: el experimento de Libet}

De manera canónica la hipótesis del experimento de Libet se enuncia de la siguiente forma: los actos voluntarios libres son precedidos por un cambio eléctrico muy específico en el cerebro llamado potencial de preparación (RP). El RP comienza $550 \mathrm{~ms}$ antes del acto y el ser humano es consciente de la intención de la acción 350-400 ms después del RP, pero $200 \mathrm{~ms}$ antes del acto motor. Lo anterior muestra que el proceso volitivo es iniciado de manera inconsciente. Sin embargó, de ello no se sigue, según Libet, que la voluntad libre no tenga

${ }^{13}$ Balconi, M. op. cit.; Roessler, J. \& N. Elian, N. op. cit. 
función alguna, sino que en la medida en que se encuentra en el lapso intermedio entre el RP y el término motor del acto, la voluntad libre ejerce una función de control del acto mediante un veto (frenar o dejar seguir el RP). ${ }^{14}$ De esta manera Libet concluye:

«The brain "decides" to initiate or, at least, to prepare to initiate the act before there is any reportable subjective awareness that such a decision has taken place», ${ }^{15}[\ldots]$ «I propose that conscious control can be exerted beforethe final motor outflow to select or control volitional outcome. The volitional process, initiated unconsciously, can either be consciously permitted to proceed toconsummation in the motor act or be consciously "vetoed."» ${ }^{16}$

La hipótesis es comprobada mediante una serie de experimentos de los cuales el siguiente es el más popular: los individuos son colocados frente a un botón el cual será presionado por estos mediante la flexión de su muñeca o dedo y que activará un punto específico en un reloj. Los individuos son monitoreados mediante un electromiógrafo para registrar la actividad eléctrica en el cerebro mientras realizan las acciones mencionadas. El individuo oprime el botón en el momento en el cual "siente" que es consciente de la intención de su acción. El tiempo de conciencia de la intención es posterior al de la actividad cerebral medida por la EMG, pero de la misma manera el tiempo de conciencia de la intención es anterior al proceso cerebral de activación motora (la actividad moto-neuronal de los músculos). ${ }^{17}$ ¿Cómo interpretar esta evidencia? Libet asegura que, a pesar de varios problemas conceptuales y técnicos, se puede concluir que el cerebro decide antes de que los agentes estén conscientes de las intenciones que conducen

${ }^{14}$ Libet, B. Unconscious Cerebral cit., pp. 529. Weǵner, D. op cit. pp.50-51, señala algunos experimentos sobre el tiempo de aparición de la conciencia previos a Libet; cf. Haggard, p. op. cit.

${ }^{15}$ Libet, B. Unconscious Cerebral cit., p. 536

${ }^{16}$ Libet, B. Unconscious Cerebral cit., p. 536-537

${ }^{17}$ Libet, B. Unconscious Cerebral cit, pp. 536-537 
al término motor del acto. ${ }^{18}$ ¿Son estas conclusiones compatibles con la negación de la agencia?

El propio Libet considera sus conclusiones limitadas para probar que la agencia es una mera ilusión. De hecho, insiste en que su mareo teórico no deja fuera a la agencia porque no puede negar la sensación o experiencia de la voluntad libre cuya evidencia se encuentra en el veto o el permiso que el agente realiza previo al acto motor. A lo mucho lo que permite su teoría es reubicar al agente en el mapa de la acción. ${ }^{19}$

Para entender cómo las conclusiones pueden o no llevar a la negación de la agencia quisiera precisar algunas relaciones conceptuales en la propuesta de Libet. En principio la acción comprende desde el RP hasta el "acto motor", es decir, el movimiento final resultante de la intención precedida del RP. La acción es el evento cronológico de los $550 \mathrm{~ms}$. Por otro lado, el aǵente no entra en la acción sino hasta los 350-400 ms después del RP y $200 \mathrm{~ms}$ antes del acto motor. Por ende, la acción total incluye cuatro elementos: el RP, la intención, el aǵente y el acto motor. ¿Cómo se relacionan estos cuatro elementos?

La hipótesis de Libet suǵiere que el inicio de la acción es llevado a cabo de manera inconsciente por el RP mientras que el control de la misma (como disparador o inhibidor) es llevado a cabo de manera consciente por el agente. Así podemos identificar tres momentos en la acción: la iniciación, la conciencia de la intención y el control de la acción. ${ }^{20}$ Los problemas comienzan a aparecer cuando se relacionan los elementos y los momentos de la acción: ¿No es la iniciación de la acción un elemento necesario para comprender la sensación de agencia? ¿En qué sentido el agente controla y está consciente de sus acciones? O en otra línea, si el cerebro puede iniciar la acción: ¿Por

${ }^{18}$ Véase, Pocket, S. \& Purdy, S. "Are Voluntary Movements Initiated Preconsciously? The Relationships between Readiness Potentials, Urges and Decisions"; Banks, W. \& Isham, E. "Do We Really Know What We Are Doinǵ? Implications Reported Time Decision for Theories of Volition", ambos en Sinnott-Armstrong \& Nadel. op. cit.

${ }^{19}$ Libet, B. Unconscious Cerebral cit. p. 536. Es probable que Libet suponga un modelo estándar de acción como el de Davidson. op. cit.; A. Roskies. "Why Libet's Studies Don't Pose a Threat to Free Will" y A. Meele. "Libet on Free Will: Rediness Potentials, Decisions, and Awareness" enSinnott-Armstrong, W. \& Nadel, L. op. cit.

${ }^{20}$ Véase, Wegner D. op. cit., pp. 29-61 
qué no puede también controlarla hasta el final? ¿No podría el cerebro realizar las otras funciones que se la atribuyen al agente?

Estas preguntas presuponen una cuestión más general: ¿̇son la iniciación, la conciencia y el control que ejerce el cerebro sobre las acciones distintos de la iniciación, conciencia y control que ejerce el agente? En lo que sigue intentaré mostrar que la iniciación, conciencia y control agencial tienen un estatus fenomenológico particular.

\section{Disrupciones en la agencia: acción y sentido de la agencia}

En la literatura sobre el SA es común citar casos anormales o "disrupciones en la agencia" para mostrar la distinción fenomenológica entre iniciación, conciencia y control del cerebro frente al "SA", es decir, la experiencia de la voluntad libre es fenomenológicamente distinta de las "acciones" que realiza el cerebro. ${ }^{21}$ En esta sección no abogaré por la contribución precisa de los casos anormales sobre los normales, ni de la relación entre varias condiciones anormales, sino que investigaré: ¿cómo las disrupciones de la agencia pueden ofrecer luz para la experiencia de SA? ${ }^{22}$

Las disrupciones en la agencia pueden ser de varios tipos. Tres de los campos más comunes para investigarlas son las patologías, los errores inconscientes y el desarrollo infantil. Me concentraré en las patologías. ${ }^{23}$ Los diversos grados en las patologías se relacionan proporcionalmente con los grados de pérdida o ausencia del SA. Así, fallos

${ }^{21}$ M. Balconi, op. cit.; Roessler, J.\& N. Elian, N. op. cit.; Wegner, D. op. cit. pp. 187270

22 Para la relación entre loseasosanormales y normales, véase, Roessler, J. \& Elian, N. "Agency and Self-Awareness: Mechanisms and Epistemology" enRoessler, J. \& Elian, N. op. cit. pp. 6-10; Levy, N. Neuroethics. Challenges for the $21^{\text {ts }}$ Century. Melbourne. Cambridge: 2007, pp. 1-67.

${ }^{23}$ Para el caso del desarrolloinfantil, veáse, Frye, D. \& Zelazo, P. "The Development of Young Children's Action Control and Awareness"; Hornsby, J. "Children's Action Control and Awareness: Comment on Frye and Zelazo" ambos en Roessler, J. \& N. Elian, N. op. cit.; Wegner, D. op. cit., pp. 151-156. Para el caso de los errores inconscientes el locus classicuses Freud, S. Zur Psychopathologie des Alltagslebens, S. Karǵer: Berlin, 1917; J. Roessler \& N. Elian, "Agency and Self-Awareness: Mechanisms and Epistemology" y Humphreys G. \& Riddoch, J. "Fractionating the Intentional Control of 


\section{El yo fracturado}

motores o de propiocepción como el síndrome de la mano anárquica revelan un fallo en la propiedad de la acción en un nivel más o menos básico (acciones corporales o acciones de pensamiento), mientras que desórdenes psicológicos generales como desorden el espectro autista, la esquizofrenia o el desorden obsesivo-compulsivo revelan un fallo en el SA en un plano más general. ${ }^{24}$ Desarrollaré tres patologías en orden progresivo relacionándolas con el SA.

La primera patología relaciona la iniciación, conciencia y el control del cuerpo con el sentido de propiedad del mismo. El síndrome de la mano anárquica (SMA) es una disfunción relacionada con el control de la acción usualmente unimanual. ${ }^{25} \mathrm{Si}$ bien las "acciones" de la mano anárquica no parecen ser tales (el sujeto se deslinda de ellas), tampoco pueden reducirse a meros reflejos. La mano afectada desempeña labores no intencionales pero complejas, bien ejecutadas y dirigidas a una meta. ${ }^{26}$ Usualmente la mano anárquica realiza la acción inversa de la mano no afectada (la mano no afectada abotona, la anárquica desabotona), aunque existen casos de acción anárquica sin presencia de una acción intencional determinada o incluso en ausencia de acción alǵuna (casos donde la mano anárquica acerca la taza de té caliente a la boca o donde la mano intenta estrangular otra persona). En cualquier caso, el paciente está consciente de los movimientos de la mano anárquica, pero es incapaz de controlarlos. ${ }^{27}$

Behaviour: A Neuropsychological Analysis", pp. 202-206, ambos en Roessler, J. \& Elian, N. op. cit.; pp. 6-10; Wegner, D. op. cit. pp. 156-166

${ }^{24}$ Véase, la sección III "Clinical Aspects Associated with Disruptions of the Sense of Agency” de Balconi, M. op. cit. Balconi agreǵa fallos en la atención (agnosias); Levy, N. op. cit. pp. 3-17

${ }^{25}$ Della Sala, S. et alia. "Righht-sidedAnarchic (Alien) Hand: A Longitudinal Study" en Neuropsychologia, 29 (11), 1991, pp. 1113-1127

${ }^{26}$ No se ha descartado por completo la presencia de intenciones inconscientes en la etiología del SMA

${ }^{27}$ Véase, Della Sala, S. op. cit. Las variedades de la sintomatología han llevado a una clasificación aún más precisa, véase, Aboitiz, F et alia. "The Alien Hand Syndrome: Classification of Forms Reported and Discussionof New Condition" en Neurol Sci, 24, 2003, pp. 252-257, quien distingue entre dispraxia diagnóstica, mano extraña, mano supernumérica y conflicto intermanual. 
¿Cómo se relaciona el síndrome de la mano anárquica con el sentido de la agencia? A diferencia de otros síndromes similares, en el SMA el paciente no niega la propiedad de la mano (síndrome de la mano extraña), sino la propiedad de las acciones realizadas (de ahí que se considere un síndrome motor y no sensorial).$^{28}$ Adicional a lo anterior el paciente está consciente de lo inapropiado de las acciones de la mano anárquica (a diferencia de la "utilización de conducta" que es altamente ambiental-dependiente).$^{29} \mathrm{El}$ paciente considera como un disturbio las acciones de la mano anárquica. ${ }^{30}$ ¿Por qué? Una probable explicación es que el agente no experimenta su iniciación, ni su control (por ello utiliza la otra mano para detener dichas acciones). Pero ¿qué hay de la consciencia? Es evidente que el agente está consciente de lo que sucede, pero lo está como un "espectador", es decir, como una tercera persona. ${ }^{31} \mathrm{El}$ inicio, conciencia y control de la acción de la mano es distinto de la "sensación" que el agente experimenta al respecto.

La segunda patología se relaciona no tanto con la propiedad de la acción particular sino con el SAen general. El trastorno obsesivo compulsivo (TOC) es un desorden de ansiedad en el cual pensamientos o imágenes intrusivos particularmente estresantes (obsesiones anormales) ${ }^{32}$ se relacionan con la urgencia de repetir actos físicos o mentales para detenerlos (compulsiones). ${ }^{33}$ Las obsesiones contienen la sensación de un error o la sensación de desastre inminente que sólo pueden

\footnotetext{
${ }^{28}$ Véase, Della Sala, S. op. cit.; Aboitiz, F. op. cit.; Marcel, A. "The Sense of Agency: Awareness and Ownership of Action" en Roessler, J \& Elian, N. op. cit. pp. 76-80 ${ }^{29}$ Véase, Della Sala S. op. cit.; cf. Lhermitte, F. et alia. "Human Autonomy and the Frontal Lobes. Part I: Imitation and Utilization Behavior: A Neuropsychological Study of 75 Patients" en Ann Neurol, 19, 1983, pp. 326-334

${ }^{30}$ Véase, Della Sala, S. op. cit.; Aboitiz, F. op. cit.; Balconi, m. op. cit. pp. 136-137,

${ }^{31}$ Véase, Marcel, A. "The Sense of Agency: Awareness and Ownership of Action" en Roessler, J. \& Elian, N. op. cit. pp. 76-80

${ }^{32}$ Rachman, S. "A Cognitive Theory of Obsessions" en Behav. Res. Ther, 35(9), 1997, pp. 793-802, señala muy bien que no toda obsesión implica necesariamente una anormalidad. Las obsesiones típicas del TOC son obsesiones anormales derivadas de una interpretación errónea y catastrófica.

${ }^{33}$ Véase, Aouizerate, et alia. "A Systematic Review of Psychosurgical Treatments for Obsessive-Compulsive Disorder: Does Deep Brain Stimulation Represent the Future
} 


\section{El yo fracturado}

ser aliviadas mediante un ritual altamente repetitivo y rígido, por ende, la sensación de responsabilidad (por el desastre) o la sensación de incompletud (por el error) son características de pacientes con TOC. ${ }^{34}$ Ambas sensaciones (de desastre o incompletitud) parecen estar relacionadas con una incongruencia entre la situación actual real y la situación interpretada por el agente (atribución excesiva de consecuencias negativas). ${ }^{35}$ En el caso de la sensación de daño el sujeto cree tener un impacto mayor sobre ciertas circunstancias del que realmente puede tener (tocarse la cabeza 20 veces para evitar que las personas mueran), mientras que en el caso de la sensación de incompletitud el sujeto cree que no ha completado una tarea de manera exitosa (revisar 25 veces si las puertas están cerradas). ${ }^{36}$

¿Qué muestra el TOC en relación con el sentido de la agencia? Paradójicamente el TOC muestra el SAde una manera desmedida (una responsabilidad excesiva) como resultado de una carencia de control sobre las obsesiones y las compulsiones. La conexión causal entre los eventos mentales y las consecuencias reales son percibidas catastróficamente. Dada la falta de control sobre situaciones usualmente fuera de nuestro control los pacientes con TOC desarrollan la ilusión de una hiper-agencia mediante la cual "recuperan" el control sobre la situación. La conciencia, el monitoreo y el control usualmente inconscientes en personas normales se tornan excesivamente explícitos en pacientes con TOC. A diferencia del SMA en el TOC el paciente está consciente de pensamientos e intenciones desde la primera persona, pero no puede ejercer control sobre ellos. Además, los pacientes

Trend in Psychosurgery?" en Clinical Neuropsychiatry, 3(6), 2006, pp. 391-403; cf. S. Rachman, R. op. cit.; Belayachi S. \& Van der Linden, M. "Looking for Outcomes: The Experience of Control and Sense of Agency in Obsessive-compulsive Behaviors" y Balconi, M. "Disruption of the Sense of Agency: From Perception to Self-Knowledge", pp. 136-137, ambos en Balconi, M. op. cit.

${ }^{34}$ La duda patológica se vincula no sólo con un aspecto cognitivo de la situación, sino con la respuesta emocional del sujeto con TOC, véase, Auoizerati, B. op. cit.

${ }^{35}$ Rachman, S. op. cit. Rachman considera clave la mala-interpretación catastrófica de los pacientes con TOG para la persistencia o disminución de los síntomas.

${ }^{36}$ Belayachi, S. \& Van der Linden, M. op. cit. pp. 157-160 
perciben tanto las obsesiones como las compulsiones como no iniciadas por ellos mismos. ${ }^{37}$

La tercera patología está vinculada con la relación entre el sujeto y la propiedad de la acción. La esquizofrenia es un trastorno en el cual el sujeto no es capaz de distinguir entre aquello que él realiza y aquello que sucede en el mundo, es decir, tiene una falta de distinción (o confusión) de los límites de la agencia y el mundo. ${ }^{38}$ Los síntomas positivos de la esquizofrenia son varios pero los más recurrentes son delirios de inserción u obstaculización de pensamientos y emociones, delirios de control, experiencias somáticas pasivas. ${ }^{39}$ La interpretación de las señales externas y su relación con el reconocimiento subjetivo suelen ser deficientes, ya sea que el paciente vincule eventos externos como producidos por él o vincule procesos internos como efectuados por un agente externo (real o ficticio). ${ }^{40}$ A diferencia del SMA el paciente con esquizofrenia no sólo se deslinda de la propiedad de la acción, sino que la proyecta o atribuye a una fuerza externa. Además, el esquizofrénico es capaz de realizar perfectamente las acciones sin intentar detenerlas (control motor), pero las experimenta como controladas por algo más. En el SMA el paciente tiene problemas con el control de la mano, mientras que en la esquizofrenia el paciente tiene problemas con la "consciencia del control de la mano." En el SMA los

${ }^{37}$ Rachman, S. op. cit.; Auoizerati, B. op. cit.; Belayachi, S. \& Van der Linden,M. op. cit. pp. 160-168

${ }^{38}$ Daprati, E. et alia. "Looking for the Agent: An Investigation into Consciousness of Action and Self Consciousness in Schizophrenic Patients" en Cognition, 65, 1997, pp. 71-86; Frith, C. "Explaining the Symptoms of Schizophrenia: Abnormalities in the Awareness of Action" enBrain Research Review, 3, 2000, pp. 357-363; Franck, N., et alia. "Defective Recognition of One's Own Actions in Schizophrenic Patients" enAmerican Journal of Psychiatry, 158, 2001, pp. 454-459; Synofzik, M. \& Voss, M. "Disturbance of the Sense of Agency in Schizophrenia" y Balcony, M. Disruption of the Sense of Agency: From Perception to Self-Knowledge", pp. 134-135 ambos en Balcony, M. op. cit.

${ }^{39}$ Frith, C. op. eit.; cf. Nicolas, F. op. cit.

${ }^{40}$ Véase, M. Synofzik\& M. Voss, "Disturbance of the Sense of Agency in Schizophrenia" y M. Balcony, "Disruption of the Sense of Agency: From Perception to SelfKnowledge", pp. 134-135 ambos enBalcony, M. op. cit.; Frith, C. op. cit. El esquizofrénico es capaz de reconocer una intención y vincularla con la acción correspondiente pero no puede asumirla como suya. Daprati, E. op. cit.; cf. Franck, N. op. cit. 


\section{El yo fracturado}

patrones de conducta de la mano son visibles, mientras que en la esquizofrenia no. ${ }^{41}$

¿Cómo se relaciona la esquizofrenia con el sentido de la aǵencia? A diferencia del SMA el paciente tiene el control motor sobre sus partes corporales, pero no es capaz de distinguir entre las acciones desarrolladas por él y las acciones de otro, de modo que sus acciones son controladas por algo más. De hecho, el paciente con esquizofrenia vincula perfectamente la intención y la acción esperada, pero no reconoce este vínculo como suyo, es decir, no siente que la acción sea suya. En cierto sentido el paciente con esquizofrenia está consciente de la acción, y la controla, pero sólo como un espectador.

¿Qué podemos concluir de todo lo anterior? La sección anterior sólo tuvo el objetivo de mostrar que existe una diferencia fenomenológica entre lo que sucede y lo que el agente "experimenta" o "siente" respecto a sus acciones. No obstante, la inferencia de la SA a la realidad de la agencia misma requiere un argumento adicional que no ofreceré. De hecho, en la siguiente sección intentaré mostrar lo contrario.

\section{4. ¿Evidencia en contra de la agencia?: Wegner y la ilusión de la agencia}

Aceptar que existe una diferencia entre lo que hace el cerebro y lo que hace y siente el agente no implica sostener el origen de la diferencia desde un punto de vista metafísico. Uno puede aceptar la diferencia fenomenológica entre lo que hace el cerebro y nuestra experiencia de la agencia e incluso sostener que es el cerebro la causa de la experiencia misma.

En su excelente obra The Illusion of Conscious Will Daniel Wegner sostiene una "teoría de la aparente causalidad de la mente", es decir, que la agencia (la voluntad consciente) es una ilusión que se gesta precisamente en el intervalo marcado por Libet entre la intención consciente y el acto motor. Un subsistema del cerebro es el encargado de dicha ilusión pues interpreta una relación causal real por una relación aparente, a saber, la relación entre el inicio inconsciente de la

${ }^{41}$ Véase, Frith, C. op. cit. 
acción y el acto motor (relación real) por la relación causal entre las intenciones del aǵente y el acto motor (relación falsa). Pero además, este subsistema nos lleva no sólo a inferir (falsamente), sino también a desarrollar la sensación de que somos nosotros a través de nuestras intenciones los que causamos las acciones cuando en el fondo nuestras acciones son fruto del automatismo, es decir, si bien la experiencia de iniciación, conciencia y control sobre nuestras acciones es diferente de la iniciación, conciencia y control real (del cerebro) esta diferencia es una ilusión creada por el cerebro mismo. ${ }^{42}$

$\mathrm{El}$ argúmento de Weǵner es progresivo. Primero muestra que existe una diferencia significativa entre la experiencia de la agencia, la causalidad de la mente y los procesos causales cerebrales. Esta diferencia es clara en la siguiente tabla de Wegner:

\begin{tabular}{|c|c|c|}
\hline & Feeling of Doing & No Feeling of Doing \\
\hline Doing & $\begin{array}{c}\text { Normal Voluntary } \\
\text { Action }\end{array}$ & Automatism \\
\hline NotDoing & Illusion of control & Normal Inaction \\
\hline
\end{tabular}

La "aparente" prueba de las acciones normales voluntarias (la causalidad del agente) se basa en la posibilidad fenomenológica de la sensación de la agencia, es decir, en la sensación de que somos nosotros mediante nuestras intenciones la causa de las acciones. Sin embargo, la voluntad consciente surge de procesos inconscientes psicológica y anatómicamente distintos de los que creemos la mente

\footnotetext{
${ }^{42}$ Weǵner, D. op. cit. pp. 1-28; unaestrategiia similar es perseguida por Haǵgard, P. "Conscious Awareness of Intention and of Action" en Roessler J. \& Elian, N. op. cit.; Wheatley, T. \& Looser, C. "Prospective Codes Fulfilled: A Potential Mechanism of Will" y T. Nadelhoffer, "The Threat of Shrinking Agency and the Free Will Disolutionism" ambos en Sinnott-Armstrong, W. \& Nadel, L. op. cit.
} 


\section{El yo fracturado}

produce. ¿Por qué sentimos entonces que somos nosotros quienes generamos nuestras acciones? Wegner afirma que la "teoría de la aparente causalidad de la mente" se basa en tres principios causales: ${ }^{43}$

Principio de prioridad: si un evento "E1" se presenta frecuentemente con anterioridad respecto a un segundo evento "E2", entonces, E1 es la causa E2.

Prioridad mental: Las intenciones se presentan frecuentemente antes que los actos, por ende, las intenciones causan los actos. ${ }^{44}$

Principio de consistencia: el efecto de una causa debe ser consistente con la causa misma.

Consistencia mental: las acciones deben ser consistentes con las intenciones que las causan. Si yo tengo la intención de tomar un vaso con agua y tomo un plato con verduras, la acción no es congruente con la intención. ${ }^{45}$

Principio de exclusividad: el efecto debe ser causado sólo y únicamente por la causa descrita.

Exclusividad mental: La acción deber ser causada únicamente por la intención descrita y no por otras causas; ya sean internas (emociones, impulsos, hábitos) o externas (otros agentes, grupos de agentes, agentes virtuales). ${ }^{46}$

La experiencia de la agencia está conformada por los tres principios. Ser un agente significa que las intenciones causan nuestras acciones, las causan de modo correcto y somos sólo nosotros (a través de ellas) los causantes. La alteración o ausencia de alguno de los tres principios repercute proporcionalmente en una disminución o desaparición de la sensación de propiedad de la acción. ¿Qué queda si

${ }^{43}$ Wegner toma los principios generales de la causalidad de Hume y los aplica a la causalidad mental, (Wegner, D. op. cit., pp. 63-70)

${ }^{44} \mathrm{E} 1$ tiempo que separa el E1 respecto de E2 no puede ser ni demasiado largo o demasiado corto como para que invalide el efecto de sucesión temporal (y por ende la inferencia causal), Weǵner, D. op. cit. pp. 70-78

${ }^{45}$ La relación consistente está compuesta por una sensación de "sin-esfuerzo" (effortless), si el efecto coincide con la causa no hay esfuerzos compensatorios para hacerlo coincidir, Wegner, D. op. cit. pp. 78-90

${ }^{46}$ Wegner, D. op. cit. pp. $90-95$ 
suprimimos los tres principios? Wegner afirma que la verdadera causalidad subyacente a los principios es el automatismo, es decir, la experiencia de la agencia es la excepción a la regla y no a la inversa, lo que tiene que ser explicado como anormal es la agencia no el automatismo. ${ }^{47}$

¿Qué es el automatismo? Negativamente el automatismo es la experiencia de la pérdida o disminución del sentido de la agencia. ${ }^{48}$ Desde un punto de vista más positivo es la generalización de los actos ideo-motores de W. James hacia una concepción ideo-motora de la acción humana. Wegner cree que el modelo de causalidad alternativo (y subyacente) al modelo de causalidad mental es el modelo ideomotor. ${ }^{49}$ En la versión de Wegner la mera idea de una acción causa de manera inmediata ( $\sin$ reflexión consciente) la acción, pero la mera idea de inacción inhibe la producción de la misma, es decir, el sistema ideomotor contiene un subsistema de control de las ideas que causan las acciones. ${ }^{50}$ Algunos autores han llamado a esta versión ideo-motora "modelo de los dos niveles" pues la acción es el resultado de la interacción entre niveles bajos de proceso de información y niveles altos de

\footnotetext{
${ }^{47}$ Wegner, D. op. cit. pp. 143-144

${ }^{48}$ Wegner ofrece la descripción de varios tipos de automatismos clásicos entre ellos están la escritura automática, la ouija, el péndulo de Chrevreul, y la radiestesia (Wegner, D. op. cit. pp. 100-120)

${ }^{49}$ William James estableció la diferencia entre acciones ideo-motoras (ideo-motor actions) y acciones por voluntad (willedactions). Las primeras se refieren a la ejecución de un movimiento a partir de la mera idea o concepción de la acción, mientras que las segundas requieren un elemento consciente adicional. El ejemplo de James es el acto de tomar conciencia del polvo en el piso y limpiarlo mientras se sigue en una conversación. La acción no supone deliberación alguna por parte del aǵente sino la mera percepción del objeto y el mero concepto fugaz del acto para realizarlo. James, W. The Principles of Psychology. New York: Henry Holt and Company, 1890, pp. 522 y ss., ef. Prinz, W. "Experimental Approaches to Action" en Roessler, J. \& Elian, N. op. cit., quien distingue entre teorías sensorimotoras e ideomotoras.

${ }^{50}$ Esta propuesta surge a partir de ciertos problemas que enfrenta la teoría. Uno de ellos es la anteriormente mencionada "utilización de la conducta" en la cual el agente no puede detener la acción que sigue de cualquier idea que venga a la mente. Si la concepción ideo-motora fuera verdadera todos los agentes padecerían de "utilización de la conducta", Wegner,D. op. cit. pp. 120-130.
} 


\section{El yo fracturado}

control de la conducta. ${ }^{51}$ De hecho la mayoría de las aproximaciones fisiológicas contemporáneas a los casos patológicos antes descritos utiliza de una u otra manera el modelo de los dos niveles para explicar la patología en cuestión. ${ }^{52}$

Si el automatismo es la regla y el SA es la excepción: ¿̇por qué el SA es tan persistente? ¿No indica esta frecuencia que el SA es la regla? Wegner reconoce que el SA es tan fuerte porque los seres humanos se conciben como un agente ideal que conoce perfectamente sus intenciones y la relación de éstas con sus acciones. ${ }^{53}$ De hecho la ilusión es tan persistente que el agente prefiere proyectar la causa de sus acciones en otras personas, grupos u otros seres (o pensar que los animales $\mathrm{y}$ objetos tienen agencia), antes que aceptar el automatismo. ${ }^{54}$

\section{Consecuencias prácticas del SA}

En esta sección final revisaré las consecuencias normativas para aceptar o rechazar la SA. La intensidad de la discusión sobre determinismo $y$ libertad ha ocasionado un reajuste de alcances $y$ consecuencias en las posturas.No obstante, intentaré establecer mi hipótesis, sobre todo, en diálogo con el trabajo de D. Pereboom quien se autodenomina "Hard-Incompatibilism" (HI) ${ }^{55}$ Las posturas clásicas en

\footnotetext{
${ }^{51}$ Véase, Roessler, J. \& Elian, N. "Agency and Self-Awareness: Mechanisms and Epistemology" enRoessler J. \& Elian, N. op. cit. pp. 6-10. Los ejemplosen la neuropsicología son Perner, J. "Dual Control and the Causal Theory of Action: The Case of Non-intentional Action" y Humphreys, G. \& Riddoch, J. "Fractionating the Intentional Control of Behaviour: A Neuropsychological Analysis" ambos en Roessler, J. \& Elian, N. op. cit. El paralelismo en la filosofía de la acción se encuentra en Frankfurt, H. op. cit.; Bratman, M. op. eit.

52 Véase, las fuentes citadas en cada caso.

${ }^{53}$ Wegner, D. op. eit. pp. 145-186

${ }^{54}$ Wegner, D. op. cit. pp. 187-316

55 Pereboom, D. Living Without Free Will. Cambridge: Cambridge University Press, 2001; Free Will, Agency, and Meaning in Life. Oxford: Oxford UniversityPress, 2014; una versión resumida se encuentra en Kane, R. op. cit. pp. 477-488. Igualmente tendré en cuenta los trabajos de Smilansky, S. Free Will and Illusion. Oxford: Oxford University Press, 2000; Strawson, G. Freedom and Belief. Oxford: Clarendon Press, 2010; y Honderich, T. How Free Are You? The Determinism Problem. New York: Oxford, 2002.
} 
torno al debate determinismo-libertad suelen tomar la siguiente forma:

Incompatibilistas: el determinismo es incompatible con el libre albedrío

Libertarios: toda acción humana es realizada libremente

Deterministas: toda acción humana está causalmente determinada

Compatibilistas: el determinismo es compatible con el libre albedrío ${ }^{56}$

Un determinista clásico sostiene tres tesis:

La libertad (en el sentido necesario para atribuir responsabilidad y mérito) es incompatible con el determinismo

No existe la libertad en este sentido, porque

Todos los eventos están determinados por causas naturales

En este sentido un determinista duro (harddeterministic) rechaza el compatibilismo (1), rechaza el libertarianismo (2) y sostiene el determinismo (3). Sin embargo, para un HI basta con sostener 1 y 2 , pues su objetivo es mostrar la implausibilidad de la existencia de la libertad entendida en modo libertario $(\neg 2)$, concepción que subyace a un compatibilista $(\neg 1)$. Pereboom afirma que la libertad presupuesta en 2 no debe ser un evento determinado por causas externas (factores causales más allá de nuestro control que determinan nuestras acciones y elecciones), ni un evento completamente fortuito (eventos producidos por nada), ni un evento parcialmente fortuito (factores fuera del control del agente producen, aunque no determinan, las acciones y elecciones).Las tres posibilidades (determinado, indeterminado total o parcial)se relacionan con el grado de control que el agente tiene de sus acciones y elecciones. ${ }^{57}$ Para un HI basta

\footnotetext{
${ }^{56}$ Hoy en día la distinción es más compleja pues en los libertarios hay aún una distinción entre "aǵent-causation" "teleological inteligibility" con variantes causales y no causales. Por razones de espacio dejaré de lado las posiciones libertarias, véase, Kane, R. op. cit. Para las dudas sobre la distinción compatibilista-incompatibilista, Smilansky, S. op. cit.; cf. Strawson, G. op. cit.; Honderich, T. op. cit.

${ }^{57}$ Pereboom, D. Living cit.; igualmente véase, Smilansky, S. op. cit. quien llama a la idea del control como moralmente central "coreconception"; Strawson, G. op. cit.
} 


\section{El yo fracturado}

con mostrar que el agente, el cual presuponen libertarios y compatibilistas tiene una existencia lo suficientemente improbable de acuerdo con las mejores explicaciones de la ciencia contemporánea. ${ }^{58}$

Cabe resaltar que las consecuencias de la negación de 1 y 2 por parte de un HI no se limitan a la responsabilidad moral (entendida como mérito), sino que impactan muchos ámbitos más como el razonamiento práctico, tratamiento criminal, significado de la vida, y las relaciones personales. ${ }^{59}$ La profundidad y gravedad del impacto depende de los argumentos ofrecidos. Así, para Dennett el impacto de la negación de 1 y 2 no es demasiado grave como para desechar la idea de la libertad en conexión con la moral, es decir, la libertad es compatible con el determinismo. ${ }^{60}$ Smilansky acepta la negación de 1 y 2 y con ello el carácter ilusorio de la libertad, pero sostiene la necesidad de esta ilusión. ${ }^{61}$ Honderich sostiene que el impacto de negar 1 y 2 es lo suficientemente grave como para considerar un mundo desconcertante y desolador. ${ }^{62}$ Por su parte Pereboom sostiene que el impacto de 1 y 2 es devastador para la noción de mérito, pero deja intactos otros conceptos centrales para la vida práctica. Incluso Pereboom afirma que el determinismo plantea una opción más viable de lo que en principio parecería. ${ }^{63}$

Bajo esta caracterización creo que mi aproximación es inmune a la crítica de Pereboom tal y como está expresada, pues el objetivo del HI es desechar la existencia de este tipo de causalidad, no la sensación de la misma. Si aceptamos que la SA es distinta de la causalidad de la mente, entonces, la validez de las premisas 1 y 2 se pone en duda. Si

${ }^{58}$ D. Pereboom. Living cit. pp. 126 y ss. El argumento de Pereboom proviene del cambio de paradiǵma en la física. Si el principio de indeterminación es verdadero, entonces, el reto a libertarios y compatibilistas se extiende no sólo si el mundo está determinado, sino también si está indeterminado. Cf. Smilansky, S. op. cit. para quien el problema con los compatibilistas radica en que su noción de libertad es teóricamente plausible, pero moralmente insuficiente; cf. Strawson, G. op. cit.

${ }^{59}$ Para una revisión detallada de las consecuencias y argumentos Pereboom, D. Living cit., pp. 127 y ss.; Pereboom, D. Free Will cit., pp. 104 y ss.

${ }^{60}$ Dennett, D. op. eit. pp. 153-172

${ }^{61}$ Smilansky, S. op. eit.

${ }^{62}$ Honderich, T. op. eit.

${ }^{63}$ Pereboom, D. Living cit., pp. 127 y ss.; Pereboom, D. Free Will cit., pp. 104 y ss. 
la libertad necesaria para atribuir responsabilidad la encontramos en $\mathrm{SA}$, y dicha libertad es plausible, entonces el SA y el determinismo son compatibles. Una mejor objeción la formularíamos en términos del propio Wegner. Tanto el determinista quien piensa que la voluntad libre es un mero proceso, como el defensor de la voluntad libre quien piensa que es un sentimiento consideran a su opuesto como equivocado inevitablemente. ${ }^{64} \mathrm{O}$ en términos de Smilansky quien piensa que el problema para el compatibilista consiste no en la plausibilidad teórica de su propuesta, sino en su insuficiencia normativa ${ }^{65}$ En ambos casos, creo que el problema que necesita ser resuelto consiste en explicar y justificar nuestra tendencia a sostener que somos libres, es decir, cómo sucede y por qué está justificada la sensación de la agencia. Sin embargo, dicha labor rebasa los propósitos de esta investigación. Cuando mucho lo aquí logrado se enmarca en la exposición de la distinción fenomenológica del SA a partir de casos patológicos.

\section{Conclusión}

La vieja diatriba entre libertad y determinismo ganó adeptos para el segundo tras los experimentos neurológicos de Libet y sus confirmaciones ulteriores. Sin embargo, el impacto de estos experimentos viró el enfoque tradicional de la casualidad de la voluntad libre o causalidad de agente hacia la noción de sensación de agencia. La SA es la sensación de iniciación, conciencia y control de las acciones a cargo del propio agente (a diferencia de la llevada por el cerebro). Si bien esta distinción fue (y sigue siendo) largamente discutida dentro de la filosofía de la mente y la acción, la discusión fenomenológica y experimental es relativamente reciente.

En la presente investigación intenté ofrecer evidencia teórico-experimental para el concepto de SA. La filosofía de la psicopatología se presentó como el terreno idóneo para este propósito. En particular discutí ampliamente algunos casos de "disrupciones en la agencia" como el SMA, el TOC y la esquizofrenia como ejemplos de la distinción

\footnotetext{
${ }^{64}$ Wegner, D. op. cit. pp. 318-325

${ }^{65}$ Smilansky, S. op. cit. 


\section{El yo fracturado}

entre estar consciente de las acciones y sentirse dueño de ellas, es decir, ejemplos de SA. Los tres ejemplos mostraron la iniciación, conciencia y control de las acciones como elementos irremplazables en la experiencia del SA, es decir, los casos patológicos entendidos como rupturas del yo reflejaron el funcionamiento idealmente óptimo del agente. Sin embargo, esta evidencia tuvo un límite probatorio, pues no consistió en una prueba de la causalidad del agente, sino en una prueba de la sensación de ésta. El límite probatorio fue establecido en concordancia con la propuesta de D. Wegner y su teoría de la aparente causalidad de la mente. El estatus ontológico del SA quedó en el nivel de una ilusión.

La defensa puntual del SA en el terreno práctico quedó lejos de los objetivos establecidos en esta investigación, lo que ofrecí en cambio fue un panorama de las consecuencias práctica en la discusión de las posturas, sobre todo a partir del HI de Pereboom. El SA es inmune a los ataques del HI tal y como están planteados actualmente. Aunque bien es cierto que mi postura deja pendiente la respuesta a otros retos como losSmilansky, y el propio Wegner, sí logró colocarse como una opción plausible.

\section{Bibliografía}

Aboitiz, Francisco et alia, (2003), "The Alien Hand Syndrome: Classification of Forms Reported and Discussion of New Condition" en Neurol Sci, 24, pp. 252-257.

Aouizerate, Bruno et alia, (2006), "A Systematic Review of Psychosurgical Treatments for Obsessive-Compulsive Disorder: Does Deep Brain Stimulation Represent the Future Trend in Psychosurgery?" en Clinical Neuropsychiatry, 3(6), pp. 391-403.

Balconi, Michaela, (ed.), (2010), Neuropsychology of the Sense of Agency. From Consciousness to Action, Springer.

Bickle, John, Mandik, Peter \& Landreth, Anthony, (2012), "The Philosophy of Neuroscience", The Stanford Encyclopedia of Philosophy, Edward N. Zalta (ed.), URL = <http://plato.stanford.edu/archives/sum2012/entries/neuroscience/>.

Bratman, Michael, (1987), Intentions, Plans, and Practical Reason, Cambridge University Press.

Block, Ned, (2007), Consciousness, Function, and Representation. Collected Papers, Vol. I, MIT Press.

Churchland, Patricia, (2011), Brain Trust. What Neuroscience Tell Us about Morality, Princeton. 
Daprati, Elena et alia, (1997), "Looking for the Agent: An Investigation into Consciousness of Action and Self Consciousness in Schizophrenic Patients" en Cognition, 65, pp. 71-86.

Davidson, Donald, (2001), Essays on Actions and Events, Oxford.

Dennett, Daniel, (1984), Elbow Room. The Varieties of Free Will Worth Wanting, Oxford.

Della Sala, Sergío et alia, (1991), "Right-sided Anarchic (Alien) Hand: A Lonǵitudinal Study" en Neuropsychologia, 29 (11), pp. 1113-1127

Franck, Nicolas, et alia, (2001), "Defective Recognition of One's Own Actions in Schizophrenic Patients" en American Journal of Psychiatry, 158, pp. 454-459

Frankfurt, Harry, (1988), The Importance of What We Care About, Cambridge.

Freud, Sigmund, (1917), Zur Psychopathologie des Alltagslebens, S. Karger

Frith, Chris, et alia, (2000), "Explaining the Symptoms of Schizophrenia: Abnormalities in the Awareness of Action" en Brain Research Review, 3, pp. 357-363

Glock, Hans-Johann, (2008), What is Analytic Philosophy?, Cambridge

Graham, George, (2010), The Disordered Mind. An Introduction to Philosophy of Mind and Mental Illness, Routledge, Taylor \& Francis. MIT

Graham, George \& Stephens Lynn (eds.), (1994), Philosophical Psychopathology,

James, William, (1890), The Principles of Psychology, London: Macmillan.

Kane, Robert, (2002), The Oxford Handbook of Free Will, New York, Oxford University Press

Kim, Jaegwon, (2000), Mind in a Physical World. An Essay on the Mind-Body Problem and Mental Causation, MIT Press

Levy, Neil, (2007), Neuroethics. Challenges for the 21ts Century, Cambridge

Libet, Benjamin, (1985), "Unconscious Cerebral Initiative and the Role of Conscious Will in Voluntary Action" en The Behavioral and Brain Sciences, 8: 529-566

Lhermitte, François, et alia, (1983), "Human Autonomy and the Frontal Lobes. Part I: Imitation and Utilization Behavior: A Neuropsychological Study of 75 Patients" en Ann Neurol, 19, pp. 326-334

Pereboom, Derk, (2001), Living without Free Will, Cambridge University Press

- , (2014), Free Will, Agency, and the Meaning of Life, Oxford University Press

Racine, Eric, (2010), Pragmatic Neuroethics. Improving Treatment and Understanding of the Mind-Brain, MIT Press

Rachman, Stanley, (1997), "A Cognitive Theory of Obsessions" en Behav. Res. Ther, 35(9), pp. 793-802

Roceo, Genaro (ed.), (2015), Disturbed Consciousness. New Essays on Psychopathology and Theories of Consciousness, The MIT Press

Roessler Johannes \& Elian Naomi (eds), (2003), Agency and Self-awareness: Issues in Philosophy and Psychology, Oxford University Press, Oxford.

Sinnott-Armstrong, Walter \& Nadel, Lynn, (eds.), (2011), Conscious Will and Responsibility. Oxford.

Smilansky, Saul, (2000), Free Will and Illusion, Oxford.

Steward, Helen \& Hyman, John, (eds.), (2004), Agency and Action, Cambridge University Press.

Strawson, Galen, (2010), Freedom and Belief, Oxford. 


\section{El yo fracturado}

Strawson, Peter, (2008), "Freedom and Resentment" en Freedom and Resentment and other Essays, Routledge, pp. 1-28.

Velleman, David, (2000), The Possibility of Practical Reason. Oxford.

Velmans, Max \& Schneider, Susan (2007), The Blackwell Companion to Consciousness, Blackwell Publishing.

Wegner, Daniel, (2002), The Illusion of Conscious Will, Cambridge, MIT Press.

Young, Garry, (2013), Philosophical Psychopathology. Philosophy without Thought Experiments, Palgrave Macmillan.

Luis Moisés López Flores lumolopez@itesm.mx 